\title{
Asymptotic Distribution of the Number of Isolated Nodes in Wireless Ad Hoc Networks With Bernoulli Nodes
}

\author{
Chih-Wei Yi, Peng-Jun Wan, Xiang-Yang Li, Member, IEEE, and Ophir Frieder, Fellow, IEEE
}

\begin{abstract}
Nodes in wireless ad hoc networks may become inactive or unavailable due to, for example, internal breakdown or being in the sleeping state. The inactive nodes cannot take part in routing/relaying, and thus may affect the connectivity. A wireless ad hoc network containing inactive nodes is then said to be connected, if each inactive node is adjacent to at least one active node and all active nodes form a connected network. This paper is the first installment of our probabilistic study of the connectivity of wireless ad hoc networks containing inactive nodes. We assume that the wireless ad hoc network consists of $n$ nodes which are distributed independently and uniformly in a unit-area disk, and are active (or available) independently with probability $p$ for some constant $0<p \leq 1$. We show that if all nodes have a maximum transmission radius $r_{n}=\sqrt{(\ln n+\xi) / \pi p n}$ for some constant $\xi$, then the total number of isolated nodes is asymptotically Poisson with mean $e^{-\xi}$, and the total number of isolated active nodes is also asymptotically Poisson with mean $p e^{-\xi}$.
\end{abstract}

Index Terms-Asymptotic distribution, Bernoulli node, isolated node, random geometric graph.

\section{INTRODUCTION}

A wireless ad hoc network is a collection of radio devices (transceivers) located in a geographic region. Each node is equipped with an omnidirectional antenna and has limited transmission power. A communication session is established either through a single-hop radio transmission if the communication parties are close enough, or through relaying by intermediate devices otherwise. Because of no need for a fixed infrastructure, wireless ad hoc networks can be flexibly deployed at low cost for varying missions, such as decision-making in the battlefield, emergency disaster relief, and environmental monitoring. In most applications, the ad hoc wireless devices are deployed

Paper approved by T. T. Lee, the Editor for Wireless Communications Theory of the IEEE Communications Society. Manuscript received August 15, 2003; revised June 18, 2005. This work was supported in part by Intel. This paper was presented in part at the IEEE Wireless Communications and Networking Conference, New Orleans, LA, March 2003.

C.-W. Yi is with the Department of Computer Science, National Chiao Tung University, Hsinchu City, Hsinchu 30010, Taiwan, R.O.C. (e-mail: yi@cs.nctu.edu.tw).

P.-J. Wan is with the Department of Computer Science, City University of Hong Kong, Hong Kong. He is also with the Department of Computer Science, Illinois Institute of Technology, Chicago, IL, USA (e-mail: pwan@cs.cityu.edu.hk; wan@cs.iit.edu).

X.-Y. Li and O. Frieder are with the Department of Computer Science, Illinois Institute of Technology, Chicago, IL, USA (e-mail: xli@cs.iit.edu; frieder@iit.edu).

Digital Object Identifier 10.1109/TCOMM.2006.869803 in a large volume. The sheer number of devices deployed, coupled with the potential harsh environment, often hinders or completely eliminates the possibility of strategic device placement, and consequently, random deployment is often the only viable option. In some other applications, the ad hoc wireless devices may be continuously in motion or be dynamically switched to on or off. For all these applications, it is natural to represent the ad hoc devices by a finite random-point process over the (finite) deployment region. Correspondingly, the wireless ad hoc network is represented by a random graph.

The classic random graph model due to Erdôs and Rényi [4], in which each pair of vertices are joined by an edge independently and uniformly at some probability, is not suited to accurately represent networks of short-range radio nodes, due to the presence of local correlation among radio links. This motivated Gilbert [5] to propose an alternative random graph model for radio networks. Gilbert's model assumes that all devices, represented by an infinite random-point process over the entire plane, have the same maximum transmission radius $r$, and two devices are joined by an edge if and only if their distance is at most $r$. For the modeling of wireless ad hoc networks which consist of finite radio nodes in a bounded geographic region, a bounded (or finite) variant of the standard Gilbert model has been used by Gupta and Kumar [6] and others. In this variant, the random-point process representing the ad hoc devices is typically assumed to be a uniform n-point process $\mathcal{X}_{n}$ over a disk or a square of unit area by proper scaling, and the wireless ad hoc network, denoted by $\mathcal{G}(n, r)$, is exactly the $r$-disk graph over $\mathcal{X}_{n}$. To distinguish the random graph $\mathcal{G}(n, r)$ from the classic random graph due to Erdôs and Rényi, it is referred to as a random geometric graph.

The connectivity of the random geometric graph $\mathcal{G}(n, r)$ has been studied by Dette and Henze [3] and Penrose [7]. For any constant $\xi$, Dette and Henze [3] showed that the graph $\mathcal{G}(n, \sqrt{(\ln n+\xi) / \pi n})$ has no isolated nodes with probability $\exp \left(-e^{-\xi}\right)$ asymptotically. Eight years later, Penrose [7] established that if a random geometric graph $\mathcal{G}(n, r)$ has no isolated nodes, then it is almost surely connected. These results are the exact analog of the counterpart in classic random graphs. However, as pointed out by Bollobás [2], we should not be misled by the remembrance: the proof for the random geometric graph is much harder.

In this paper, we consider an extension to the random geometric graph $\mathcal{G}(n, r)$ by introducing an additional assumption 
that all nodes are active (or available) independently with probability $p$ for some constant $0<p \leq 1$. Such extension is motivated by the fault tolerance of wireless ad hoc networks. In a practical wireless ad hoc network, a node may be inactive (or unavailable) due to either internal breakdown, or being in the sleeping state. In either case, the inactive nodes will not take part in routing/relaying, and thus may affect the connectivity. It is natural to model the availability of the nodes by a Bernoulli model, and hence, we call the nodes Bernoulli nodes. A wireless ad hoc network of Bernoulli nodes is then said to be connected if each inactive node is adjacent to at least one active node, and all active nodes form a connected network.

Our probabilistic study of the connectivity of the random geometric graph with Bernoulli nodes consists of two installments due to the lengthy analysis. The first installment, which is the focus of this paper, addresses the distribution of the number of nodes without active neighbors. For convenience, a node is said to be isolated from active nodes, or simply isolated, if it has no active neighbors. We shall prove that both the number of isolated nodes and the number of isolated active nodes have asymptotic Poisson distributions. The second installment, which will be reported in a separate paper, proves that if a random geometric graph with Bernoulli nodes has no isolated nodes, it is also connected almost surely.

In what follows, $\|x\|$ is the Euclidean norm of a point $x \in \mathbb{R}^{2}$, and $|A|$ is shorthand for the 2-D Lebesgue measure (or area) of a measurable set $A \subset \mathbb{R}^{2}$. All integrals considered will be Lebesgue integrals. The topological boundary of a set $A \subset \mathbb{R}^{2}$ is denoted by $\partial A$. The disk of radius $r$ centered at $x$ is denoted by $D(x, r)$. The special unit-area disk centered at the origin is denoted by $\Omega$. For any set $S$ and positive integer $k$, the $k$-fold Cartesian product of $S$ is denoted by $S^{k}$. The symbols $O, o$, and $\sim$ always refer to the limit $n \longrightarrow \infty$. To avoid trivialities, we tacitly assume $n$ to be sufficiently large if necessary. For simplicity of notation, the dependence of sets and random variables on $n$ will be frequently suppressed.

The remainder of this paper is organized as follows. In Section II, we present several useful geometric results and integrals. In Section III, we derive both the distribution of the number of isolated nodes and the distribution of the number of isolated active nodes. In Section IV, we give a short summary and show future work.

\section{GEOMETRY OF DISKS}

The results in this section are purely geometric, with no probabilistic content. Let $r$ be the transmission radius of the nodes. For any finite set of nodes $\left\{x_{1}, \ldots, x_{k}\right\}$ in $\Omega$, we use $G_{r}\left(x_{1}, \ldots, x_{k}\right)$ to denote the graph over $\left\{x_{1}, \ldots, x_{k}\right\}$, in which there is an edge between two nodes if and only if their Euclidean distance is at most $r$. For any positive integers $k$ and $m$ with $1 \leq m \leq k$, let $C_{k m}$ denote the set of $\left(x_{1}, \ldots, x_{k}\right) \in \Omega^{k}$ satisfying that $G_{2 r}\left(x_{1}, \ldots, x_{k}\right)$ has exactly $m$ connected components.

We partition the unit-area disk $\Omega$ into three regions, $\Omega(0)$, $\Omega(1)$, and $\Omega(2)$, as shown in Fig. $1: \Omega(0)$ is the disk of radius $1 / \sqrt{\pi}-r$ centered at the origin; $\Omega(1)$ is the annulus of radii

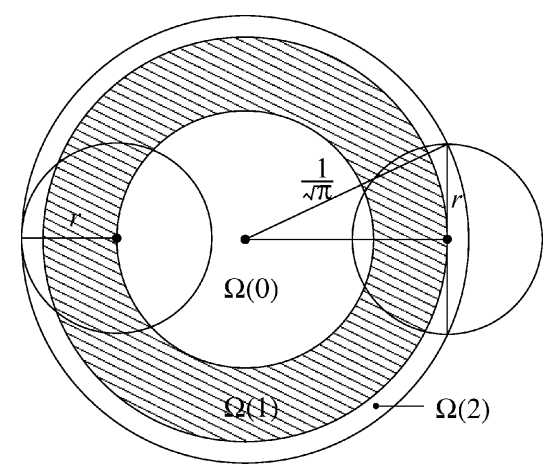

Fig. 1. Partition of the unit-area disk $\Omega$.

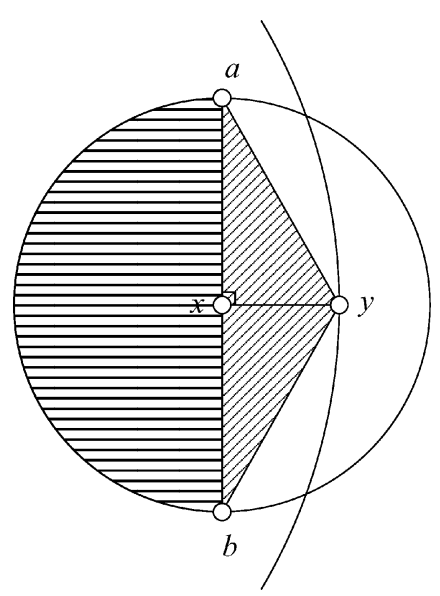

Fig. 2. Half-disk and the triangle.

$1 / \sqrt{\pi}-r$ and $\sqrt{1 / \pi-r^{2}}$ centered at the origin; and $\Omega(2)$ is the annulus of radii $\sqrt{1 / \pi-r^{2}}$ and $1 / \sqrt{\pi}$ centered at the origin. Then

$$
\begin{aligned}
& |\Omega(0)|=(1-\sqrt{\pi} r)^{2} \\
& |\Omega(1)|=2 \pi r\left(\frac{1}{\sqrt{\pi}-r}\right) \\
& |\Omega(2)|=\pi r^{2} .
\end{aligned}
$$

For any set $S \subseteq \Omega$ and $r>0$, the $r$-neighborhood of $S$ is the set $\bigcup_{x \in S} D(x, r) \cap \Omega$. We use $\nu_{r}(S)$ to denote the area of the $r$-neighborhood of $S$, and sometimes by slightly abusing the notation, to denote the $r$-neighborhood of $S$ itself. Obviously, for any $x \in \Omega, \nu_{r}(x) \geq \pi r^{2} / 3$. If $x \in \Omega(0), \nu_{r}(x)=\pi r^{2}$. If $x \in \Omega(1)$, we have the following tighter lower bound on $\nu_{r}(x)$.

Lemma 1: For any $x \in \Omega(1)$

$$
\nu_{r}(x) \geq \frac{\pi r^{2}}{2}+\left(\frac{1}{\sqrt{\pi}}-\|x\|\right) r .
$$

Proof: Let $y$ be the point in $\partial \Omega$ such that $\|y-x\|=$ $(1 / \sqrt{\pi})-\|x\|$, and $a b$ be the diameter of $D(x, r)$ perpendicular to $x y$ (see Fig. 2). Then $\nu_{r}(x)$ contains a half-disk of $D(x, r)$ to the side of $a b$ opposite to $y$, and the triangle $a b y$. Since the area of the triangle $a b y$ is exactly $((1 / \sqrt{\pi})-\|x\|) r$, the lemma follows.

The next lemma gives a lower bound on the area of the $r$-neighborhood of more than one node. 


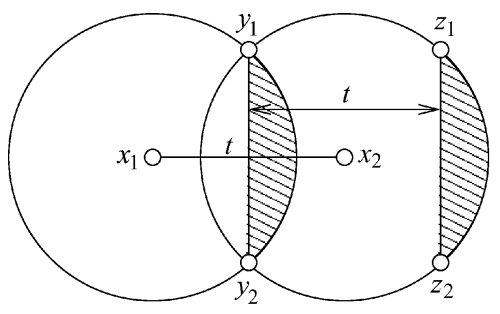

(a)

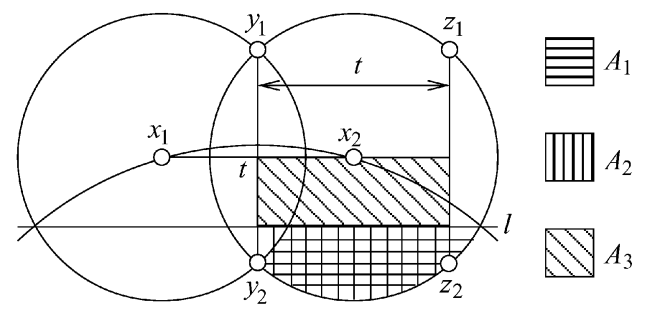

(b)

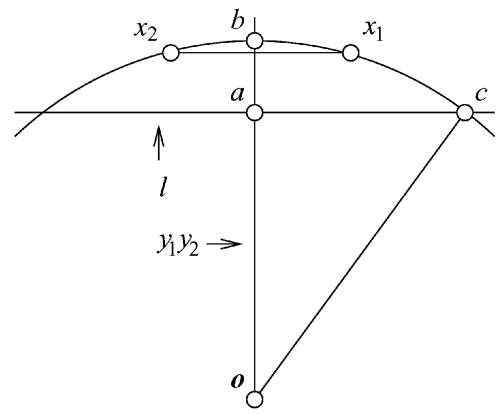

(c)

Fig. 3. Area of two intersecting disks.

Lemma 2: Assume that

$$
r \leq \frac{\frac{1}{\sqrt{\pi}}}{\frac{12}{\pi}+\frac{\pi}{12}} \approx \frac{0.245}{\sqrt{\pi}}
$$

Let $x_{1}, \ldots, x_{k}$ be a sequence of $k \geq 2$ nodes in $\Omega$, such that $x_{1}$ has the largest norm, and $\left\|x_{i}-x_{j}\right\| \leq 2 r$ if and only if $|i-j| \leq 1$. Then

$$
\nu_{r}\left(x_{1}, \ldots, x_{k}\right) \geq \nu_{r}\left(x_{1}\right)+\frac{\pi}{12} r \sum_{i=1}^{k-1}\left\|x_{i+1}-x_{i}\right\| .
$$

Proof: We prove the lemma by induction on $k$. We begin with $k=2$. Let $t=\left\|x_{2}-x_{1}\right\|$ and $f(t)=\left|D\left(x_{2}, r\right) \backslash D\left(x_{1}, r\right)\right|$. We first show that $f(t) \geq(\pi / 2) r t$. Let $y_{1} y_{2}$ be the common chord of $\partial D\left(x_{1}, r\right)$ and $\partial D\left(x_{2}, r\right)$, and let $z_{1} z_{2}$ be another chord of $\partial D\left(x_{2}, r\right)$ that is parallel to $y_{1} y_{2}$ and has the same length as $y_{1} y_{2}$ [see Fig. 3(a)]. Then $f(t)$ is also equal to the area of the portion of $D\left(x_{2}, r\right)$ between the two chords $y_{1} y_{2}$ and $z_{1} z_{2}$. Thus, $f^{\prime}(t)=\left\|y_{1} y_{2}\right\|$, which is decreasing over [0,2r]. Therefore, $f(t)$ is concave over $[0,2 r]$. Since $f(0)=0$ and $f(2 r)=\pi r^{2}$, we have $f(t) \geq(\pi / 2) r t$.

Now we are ready to prove the lemma for $k=2$. If $x_{1} \in \Omega(0)$, then $\nu_{r}\left(x_{1}, x_{2}\right)-\nu_{r}\left(x_{1}\right)$ is exactly $f(t)$, and thus, the lemma follows immediately from $f(t) \geq(\pi / 2) r t$. So we assume that $x_{1} \notin \Omega(0)$. Note that for the same distance $t$, $\nu_{r}\left(x_{1}, x_{2}\right)-\nu_{r}\left(x_{1}\right)$ achieves its minimum when both $x_{1}$ and $x_{2}$ are in $\partial \Omega$. It is sufficient to prove the lemma for $x_{1}, x_{2} \in \partial \Omega$.
Let $y_{1} y_{2}$ and $z_{1} z_{2}$ be the two chords of $\partial D\left(x_{2}, r\right)$ as above, with $y_{2} \in \Omega$, and $\ell$ be the line through the two intersection points between $\partial \Omega$ and $\partial\left(D\left(x_{1}, r\right) \cup D\left(x_{2}, r\right)\right)$ [see Fig. 3(b)]. We use $A_{1}$ to denote the portion of $D\left(x_{2}, r\right) \backslash D\left(x_{1}, r\right)$ which lies in the same side of $\ell$ as $y_{2}$; use $A_{2}$ to denote the portion of $D\left(x_{2}, r\right)$ which is surrounded by $y_{1} y_{2}, z_{1} z_{2}, \ell$, and the short arc between $y_{2}$ and $z_{2}$; and use $A_{3}$ to denote the rectangle surrounded by $y_{1} y_{2}, z_{1} z_{2}, \ell$, and the line through $x_{1}$ and $x_{2}$. Then

$$
\nu_{r}\left(x_{1}, x_{2}\right)-\nu_{r}\left(x_{1}\right) \geq\left|A_{1}\right|=\left|A_{2}\right|=\frac{f(t)}{2}-\left|A_{3}\right| .
$$

An upper bound on $\left|A_{3}\right|$ can be obtained as follows. Let $a$ be the intersection point between $y_{1} y_{2}$ and $\ell, b$ be the intersection point between $y_{1} y_{2}$ and $\partial \Omega$, and $c$ be an intersection point between $\ell$ and $\partial \Omega$ [see Fig. 3(c)]. Then $\|a c\| \leq 2 r$, and

$$
\|o a\|=\sqrt{\|o c\|^{2}-\|a c\|} \geq \sqrt{\frac{1}{\pi}-(2 r)^{2}} .
$$

Hence

$$
\begin{aligned}
\|a b\| & =\|o b\|-\|o a\| \\
& \leq \frac{1}{\sqrt{\pi}}-\sqrt{\frac{1}{\pi}-(2 r)^{2}} \\
& =\frac{4 r^{2}}{\frac{1}{\sqrt{\pi}}+\sqrt{\frac{1}{\pi}-(2 r)^{2}}} .
\end{aligned}
$$

Note that one side of $A_{3}$ is exactly $t$, and the other side is at most $\|a b\|$. Thus

$$
\left|A_{3}\right| \leq \frac{4 r^{2} t}{\frac{1}{\sqrt{\pi}}+\sqrt{\frac{1}{\pi}-(2 r)^{2}}} .
$$

As $f(t) \geq(\pi / 2) r t$, we have

$$
\nu_{r}\left(x_{1}, x_{2}\right)-\nu_{r}\left(x_{1}\right) \geq\left(\frac{\pi}{4}-\frac{4 r}{\frac{1}{\sqrt{\pi}}+\sqrt{\frac{1}{\pi}-(2 r)^{2}}}\right) r t .
$$

It is straightforward to verify that if

$$
r \leq \frac{\frac{1}{\sqrt{\pi}}}{\frac{12}{\pi}+\frac{\pi}{12}} \approx \frac{0.245}{\sqrt{\pi}}
$$

then

$$
\frac{\pi}{4}-\frac{4 r}{\frac{1}{\sqrt{\pi}}+\sqrt{\frac{1}{\pi}-(2 r)^{2}}} \geq \frac{\pi}{12}
$$

and thereby the lemma for $k=2$ follows.

In the following, we assume the lemma is true for at most $k-1$ nodes, and we shall show that the lemma is true for $k$ nodes. If $k=3$, then

$$
\begin{aligned}
& \nu_{r}\left(x_{1}, x_{2}, x_{3}\right) \geq \nu_{r}\left(x_{1}\right)+\nu_{r}\left(x_{3}\right) \geq \nu_{r}\left(x_{1}\right)+\frac{\pi r^{2}}{3} \\
& =\nu_{r}\left(x_{1}\right)+\frac{\pi}{12} r \cdot 4 r \geq \nu_{r}\left(x_{1}\right)+\frac{\pi}{12} r \sum_{i=1}^{2}\left\|x_{i+1}-x_{i}\right\| .
\end{aligned}
$$


If $k>3$, then by the inductions hypothesis

$$
\begin{aligned}
\nu_{r}\left(x_{1}, \ldots, x_{k}\right) & \geq \nu_{r}\left(x_{1}, \ldots, x_{k-2}\right)+\nu_{r}\left(x_{k}\right) \\
& \geq \nu_{r}\left(x_{1}\right)+\frac{\pi}{12} r \sum_{i=1}^{k-3}\left\|x_{i+1}-x_{i}\right\|+\frac{\pi r^{2}}{3} \\
& \geq \nu_{r}\left(x_{1}\right)+\frac{\pi}{12} r \sum_{i=1}^{k-1}\left\|x_{i+1}-x_{i}\right\| .
\end{aligned}
$$

Therefore, the lemma is true by induction.

Corollary 3: Assume that

$$
r \leq \frac{\frac{1}{\sqrt{\pi}}}{\frac{12}{\pi}+\frac{\pi}{12}} \approx \frac{0.245}{\sqrt{\pi}} .
$$

Then for any $\left(x_{1}, \ldots, x_{k}\right) \in C_{k 1}$ with $x_{1}$ being the one of the largest norm among $x_{1}, \ldots, x_{k}$

$$
\nu_{r}\left(x_{1}, \ldots, x_{k}\right) \geq \nu_{r}\left(x_{1}\right)+\frac{\pi}{12} r \max _{2 \leq i \leq k}\left\|x_{i}-x_{1}\right\| .
$$

Proof: Without loss of generality, we assume that $\| x_{k}-$ $x_{1} \|$ achieves $\max _{2 \leq i \leq k}\left\|x_{i}-x_{1}\right\|$. Let $P$ be a min-hop path between $x_{1}$ and $x_{k}$ in $\left.G_{2 r} \leq x_{1}, x_{2}, \ldots, x_{k}\right)$ and $t$ be the total length of $P$. Then every pair of nodes in $P$ that are not adjacent nodes in $P$ are separated by a distance of more than $2 r$. Thus, by applying Lemma 2 to the nodes in $P$, we obtain

$$
\nu_{r}\left(\left\{x_{i} \mid x_{i} \in P\right\}\right) \geq \nu_{r}\left(x_{1}\right)+\frac{\pi}{12} r t .
$$

Since $\nu_{r}\left(x_{1}, \ldots, x_{k}\right) \geq \nu_{r}\left(\left\{x_{i} \mid x_{i} \in P\right\}\right)$ and $t \geq\left\|x_{k}-x_{1}\right\|$, the corollary follows.

In the remaining of this section, we give the limits of several integrals.

Lemma 4: For any $z \in[0,1 / 2], e^{-z-z^{2}} \leq 1-z \leq e^{-z}$.

Proof: For any $z \geq 0,1-z \leq e^{-z} \leq 1-z+\left(z^{2} / 2\right)$. If $z \in[0,1 / 2]$, then

$$
\begin{aligned}
e^{-z-z^{2}} & \leq\left(1-z+\frac{z^{2}}{2}\right)\left(1-z^{2}+\frac{z^{4}}{2}\right) \\
& =1-z-z^{2}\left(\frac{1}{2}-z\right)-\frac{1}{4} z^{5}(2-z) \leq 1-z
\end{aligned}
$$

Lemma 5: Let $r=\sqrt{(\ln n+\xi) / \pi p n}$ for some constant $\xi$. Then

$$
\begin{aligned}
n \int_{\Omega} e^{-n p \nu_{r}(x)} d x & \sim e^{-\xi}, \\
n \int_{\Omega}\left(1-p \nu_{r}(x)\right)^{n-1} d x & \sim e^{-\xi} .
\end{aligned}
$$

Proof: We only give the proof of the first asymptotic equality. The second one can be proved in the similar manner together with the inequalities in Lemma 4. First, we calculate the integration over $\Omega(0)$

$$
n \int_{\Omega(0)} e^{-n p \nu_{r}(x)} d x=n e^{-n p \pi r^{2}}|\Omega(0)| \sim n e^{-n p \pi r^{2}}=e^{-\xi} .
$$

Now, we calculate the integration over $\Omega(2)$

$$
\begin{aligned}
n \int_{\Omega(2)} e^{-n p \nu_{r}(x)} d x & \leq n e^{-\frac{1}{3} n p \pi r^{2}}|\Omega(2)| \\
& =n \pi r^{2} e^{-\frac{1}{3} n p \pi r^{2}}=o(1) .
\end{aligned}
$$

Next, we calculate the integration over $\Omega(1)$. By Lemma 1

$$
n \int_{\Omega(1)} e^{-n p \nu_{r}(x)} d x
$$$$
\leq n e^{-\frac{n p \pi r^{2}}{2}} \int_{\Omega(1)} e^{-n p r\left(\frac{1}{\sqrt{\pi}}-\|x\|\right)} d x
$$$$
=2 \pi n e^{-\frac{n p \pi r^{2}}{2}} \int_{\frac{1}{\sqrt{\pi}}-r}^{\sqrt{\frac{1}{\pi}-r^{2}}} \rho e^{-n p r\left(\frac{1}{\sqrt{\pi}}-\rho\right)} d \rho
$$$$
\leq 2 \pi n e^{-\frac{n p \pi r^{2}}{2}} \int_{\frac{1}{\sqrt{\pi}}-r}^{\frac{1}{\sqrt{\pi}}} \rho e^{-n p r\left(\frac{1}{\sqrt{\pi}}-\rho\right)} d \rho
$$$$
\leq 2 \sqrt{\pi} n e^{-\frac{n p \pi r^{2}}{2}} \int_{\frac{1}{\sqrt{\pi}}-r}^{\frac{1}{\sqrt{\pi}}} e^{-n p r\left(\frac{1}{\sqrt{\pi}}-\rho\right)} d \rho
$$$$
=2 \sqrt{\pi} n e^{-\frac{n p \pi r^{2}}{2}} \int_{0}^{r} e^{-n p r t} d t
$$

$$
\leq \frac{2 \sqrt{\pi}}{p} \frac{1}{r} e^{-\frac{n p \pi r^{2}}{2}}=O(1)(\ln n)^{-\frac{1}{2}}=o(1) .
$$

Therefore

$$
n \int_{\Omega} e^{-n p \nu_{r}(x)} d x \sim e^{-\xi}
$$

Lemma 6: Let $r=\sqrt{(\ln n+\xi) / \pi p n}$ for some constant $\xi$. Then for any fixed integer $k \geq 2$

$$
\begin{array}{r}
n^{k} \int_{C_{k 1}} e^{-n p \nu_{r}\left(x_{1}, x_{2}, \ldots, x_{k}\right)} \prod_{i=1}^{k} d x_{i}=o(1) \\
n^{k} \int_{C_{k 1}}\left(1-p \nu_{r}\left(x_{1}, x_{2}, \ldots, x_{k}\right)\right)^{n-k} \prod_{i=1}^{k} d x_{i}=o(1) .
\end{array}
$$

Proof: Since

$$
\left(1-p \nu_{r}\left(x_{1}, x_{2}, \cdots, x_{k}\right)\right)^{n-k} \leq \frac{e^{-n p \nu_{r}\left(x_{1}, x_{2}, \ldots, x_{k}\right)}}{\left(1-p k \pi r^{2}\right)^{k}}
$$

the second equality would follow from the first one. Hence, we only have to prove the first one. Let $S$ denote the set of $\left(x_{1}, x_{2}, \ldots, x_{k}\right) \in C_{k 1}$ satisfying that $x_{1}$ is the one with largest norm among $x_{1}, \ldots, x_{k}$, and $x_{2}$ is the one with longest distance from $x_{1}$ among $x_{2}, \ldots, x_{k}$. Then

$$
\begin{aligned}
n^{k} \int_{C_{k 1}} e^{-n p \nu_{r}\left(x_{1}, x_{2}, \ldots, x_{k}\right)} \prod_{i=1}^{k} d x_{i} \\
\leq k(k-1) n^{k} \int_{S} e^{-n p \nu_{r}\left(x_{1}, x_{2}, \ldots, x_{k}\right)} \prod_{i=1}^{k} d x_{i} .
\end{aligned}
$$


So it suffices to prove

$$
n^{k} \int_{S} e^{-n p \nu_{r}\left(x_{1}, x_{2}, \ldots, x_{k}\right)} \prod_{i=1}^{k} d x_{i}=o(1) .
$$

Note that for any $\left(x_{1}, x_{2}, \ldots, x_{k}\right) \in S$

$$
\nu_{r}\left(x_{1}\right)+c r\left\|x_{2}-x_{1}\right\| \leq \nu_{r}\left(x_{1}, x_{2}, \ldots, x_{k}\right) \leq k \pi r^{2}
$$

for some constant $c$ by Corollary 3 , and

$$
\begin{aligned}
& x_{i} \in B\left(x_{1},\left\|x_{2}-x_{1}\right\|\right), \quad 3 \leq i \leq k \\
& x_{2} \in B\left(x_{1}, 2(k-1) r\right) .
\end{aligned}
$$

Thus

$$
\begin{aligned}
& n^{k} \int_{S} e^{-n p \nu_{r}\left(x_{1}, x_{2}, \ldots, x_{k}\right)} \prod_{i=1}^{k} d x_{i} \\
& \leq n^{k} \int_{S} e^{-n p\left(\nu_{r}\left(x_{1}\right)+c r\left\|x_{2}-x_{1}\right\|\right)} \prod_{i=1}^{k} d x_{i} \\
& \leq n^{k} \int_{\Omega} e^{-n p \nu_{r}\left(x_{1}\right)} d x_{1} \int_{B\left(x_{1}, 2(k-1) r\right)} e^{-n p c r\left\|x_{2}-x_{1}\right\|} d x_{2} \\
& \cdot \prod_{i=3}^{k} \int_{B\left(x_{1},\left\|x_{2}-x_{1}\right\|\right)} d x_{i} \\
& =n^{k} \int_{\Omega} e^{-n p \nu_{r}\left(x_{1}\right)} d x_{1} \\
& \text {. } \int_{B\left(x_{1}, 2(k-1) r\right)} e^{-n p c r\left\|x_{2}-x_{1}\right\|}\left(\pi\left\|x_{2}-x_{1}\right\|^{2}\right)^{k-2} d x_{2} \\
& =2 \pi^{k-1}\left(n \int_{\Omega} e^{-n p \nu_{r}\left(x_{1}\right)} d x_{1}\right) \\
& \cdot\left(n^{k-1} \int_{0}^{2(k-1) r} e^{-n p c r \rho} \rho^{2 k-3} d \rho\right) \\
& <2 \pi^{k-1}\left(n \int_{\Omega} e^{-n p \nu_{r}\left(x_{1}\right)} d x_{1}\right) \\
& \cdot\left(n^{k-1} \int_{0}^{\infty} e^{-n p c r \rho} \rho^{2 k-3} d \rho\right) \\
& =\frac{(2 k-3) ! 2 \pi^{k-1} n^{k-1}}{(n p c r)^{2 k-2}}\left(n \int_{\Omega} e^{-n p \nu_{r}\left(x_{1}\right)} d x_{1}\right) \\
& =O(1) \frac{n \int_{\Omega} e^{-n p \nu_{r}\left(x_{1}\right)} d x_{1}}{(\ln n)^{k-1}}=o(1)
\end{aligned}
$$

where the last equality follows from Lemma 5 .

Lemma 7: Let $r=\sqrt{(\ln n+\xi) / \pi p n}$ for some constant $\xi$. Then for any fixed integers $2 \leq m<k$

$$
\begin{array}{r}
n^{k} \int_{C_{k m}} e^{-n p \nu_{r}\left(x_{1}, x_{2}, \ldots, x_{k}\right)} \prod_{i=1}^{k} d x_{i}=o(1) \\
n^{k} \int_{C_{k m}}\left(1-p \nu_{r}\left(x_{1}, x_{2}, \ldots, x_{k}\right)\right)^{n-k} \prod_{i=1}^{k} d x_{i}=o(1) .
\end{array}
$$

Proof: Since

$$
\left(1-p \nu_{r}\left(x_{1}, x_{2}, \ldots, x_{k}\right)\right)^{n-k} \leq \frac{e^{-n p \nu_{r}\left(x_{1}, x_{2}, \ldots, x_{k}\right)}}{\left(1-p k \pi r^{2}\right)^{k}}
$$

the second equality would follow from the first one, and thus we only have to prove the first one. For any $m$-partition $\Pi=$ $\left\{K_{1}, K_{2}, \ldots, K_{m}\right\}$ of $\{1,2, \ldots, k\}$, let $\Omega^{k}(\Pi)$ denote the set of $\left(x_{1}, x_{2}, \ldots, x_{k}\right) \in \Omega^{k}$, such that for any $1 \leq j \leq m$, the nodes $\left\{x_{i}: i \in K_{j}\right\}$ form a connected component of $G_{2 r}\left(x_{1}, x_{2}, \ldots, x_{k}\right)$. Then $C_{k m}$ is the union of $\Omega^{k}(\Pi)$ over all $m$-partitions $\Pi$ of $\{1,2, \ldots, k\}$. So it is sufficient to show that for any $m$-partition $\Pi$ of $\{1,2, \ldots, k\}$

$$
n^{k} \int_{\Omega^{k}(\Pi)} e^{-n p \nu_{r}\left(x_{1}, x_{2}, \ldots, x_{k}\right)} \prod_{i=1}^{k} d x_{i}=o(1) .
$$

Now fix an $m$-partition $\Pi=\left\{K_{1}, K_{2}, \ldots, K_{m}\right\}$ of $\{1,2, \ldots, k\}$, and let $l_{j}=\left|K_{j}\right|$ for $1 \leq j \leq m$. Then

$$
\Omega^{k}(\Pi) \subseteq \prod_{j=1}^{m} C_{l_{j} 1}
$$

and for any $\left(x_{1}, x_{2}, \ldots, x_{k}\right) \in \Omega^{k}(\Pi)$

$$
\nu_{r}\left(x_{1}, x_{2}, \ldots, x_{k}\right)=\sum_{j=1}^{m} \nu_{r}\left(\left\{x_{i} \mid i \in K_{j}\right\}\right) .
$$

Thus

$$
\begin{aligned}
& n^{k} \int_{\Omega^{k}(\Pi)} e^{-n p \nu_{r}\left(x_{1}, x_{2}, \ldots, x_{k}\right)} \prod_{i=1}^{k} d x_{i} \\
& =n^{k} \int_{\Omega^{k}(\Pi)} e^{-n p \sum_{j=1}^{m} \nu_{r}\left(\left\{x_{i} \mid i \in K_{j}\right\}\right)} \prod_{i=1}^{k} d x_{i} \\
& =n^{k} \int_{\Omega^{k}(\Pi)} \prod_{j=1}^{m} e^{-n p \nu_{r}\left(\left\{x_{i} \mid i \in K_{j}\right\}\right)} \prod_{i=1}^{k} d x_{i} \\
& \leq n^{k} \prod_{j=1}^{m} \int_{C_{l_{j} 1}} e^{-n p \nu_{r}\left(\left\{x_{i} \mid i \in K_{j}\right\}\right)} \prod_{i \in K_{j}} d x_{i} \\
& =\prod_{j=1}^{m}\left(n^{l_{j}} \int_{C_{l_{j} 1}} e^{-n p \nu_{r}\left(\left\{x_{i} \mid i \in K_{j}\right\}\right)} \prod_{i \in K_{j}} d x_{i}\right)=o(1)
\end{aligned}
$$

where the last equality follows from Lemma 6 , and the fact that at least one $l_{j} \geq 2$.

Lemma 8: Let $r=\sqrt{(\ln n+\xi) / \pi p n}$ for some constant $\xi$. Then for any fixed integer $k \geq 2$

$$
\begin{aligned}
n^{k} \int_{C_{k k}} e^{-n p \nu_{r}\left(x_{1}, x_{2}, \ldots, x_{k}\right)} \prod_{i=1}^{k} d x_{i} & \sim e^{-k \xi} \\
n^{k} \int_{C_{k k}}\left(1-p \nu_{r}\left(x_{1}, x_{2}, \ldots, x_{k}\right)\right)^{n-k} \prod_{i=1}^{k} d x_{i} & \sim e^{-k \xi} .
\end{aligned}
$$

Proof: We again only give the proof of the first asymptotic equality and remark that the second one can be proved in a sim- 
ilar manner, together with the inequalities in Lemma 4. For any $\left(x_{1}, x_{2}, \ldots, x_{k}\right) \in C_{k k}$

$$
\nu_{r}\left(x_{1}, x_{2}, \ldots, x_{k}\right)=\sum_{i=1}^{k} \nu_{r}\left(x_{i}\right) .
$$

Thus

$$
\begin{aligned}
& n^{k} \int_{C_{k k}} e^{-n p \nu_{r}\left(x_{1}, x_{2}, \ldots, x_{k}\right)} \prod_{i=1}^{k} d x_{i} \\
& =n^{k} \int_{C_{k k}} e^{-n p \sum_{i=1}^{k} \nu_{r}\left(x_{i}\right)} \prod_{i=1}^{k} d x_{i} \\
& =n^{k} \int_{\Omega^{k}} e^{-n p \sum_{i=1}^{k} \nu_{r}\left(x_{i}\right)} \prod_{i=1}^{k} d x_{i} \\
& -n^{k} \int_{\Omega^{k} \backslash C_{k k}} e^{-n p \sum_{i=1}^{k} \nu_{r}\left(x_{i}\right)} \prod_{i=1}^{k} d x_{i} .
\end{aligned}
$$

We show the first term is asymptotically equal to $e^{-k \xi}$, and the second term is asymptotically negligible. Indeed

$$
\begin{aligned}
& n^{k} \int_{\Omega^{k}} e^{-n p \sum_{i=1}^{k} \nu_{r}\left(x_{i}\right)} \prod_{i=1}^{k} d x_{i} \\
& \quad=n^{k} \int_{\Omega^{k}}^{k} \prod_{i=1}^{-n p \nu_{r}\left(x_{i}\right)} \prod_{i=1}^{k} d x_{i} \\
& \quad=\prod_{i=1}^{k}\left(n \int_{\Omega} e^{-n p \nu_{r}\left(x_{i}\right)} d x_{i}\right) \sim e^{-k \xi}
\end{aligned}
$$

where the last equality follows from Lemma 5 . Note that for any $\left(x_{1}, x_{2}, \ldots, x_{k}\right) \in \Omega^{k} \backslash C_{k k}$

$$
\nu_{r}\left(x_{1}, x_{2}, \ldots, x_{k}\right) \leq \sum_{i=1}^{k} \nu_{r}\left(x_{i}\right) .
$$

Thus

$$
\begin{aligned}
& n^{k} \int_{\Omega^{k} \backslash C_{k k}} e^{-n p \sum_{i=1}^{k} \nu_{r}\left(x_{i}\right)} \prod_{i=1}^{k} d x_{i} \\
& \leq n^{k} \int_{\Omega^{k} \backslash C_{k k}} e^{-n p \nu_{r}\left(x_{1}, x_{2}, \ldots, x_{k}\right)} \prod_{i=1}^{k} d x_{i} \\
& =\sum_{m=1}^{k-1} n^{k} \int_{C_{k m}} e^{-n p \nu_{r}\left(x_{1}, x_{2}, \ldots, x_{k}\right)} \prod_{i=1}^{k} d x_{i}=o(1)
\end{aligned}
$$

where the last equality follows from Lemmas 6 and 7.

\section{ASYMPTOTIC DistribUtion OF THE NUMBER OF ISOLATED NODES}

The main result of this paper is the following theorem.
Theorem 9: Suppose that all nodes have a maximum transmission radius $r=\sqrt{(\ln n+\xi) / \pi p n}$ for some constant $\xi$. Then the total number of isolated nodes is asymptotically Poisson with mean $e^{-\xi}$, and the total number of isolated active nodes is also asymptotically Poisson with mean $p e^{-\xi}$.

The above theorem will be proved by using Brun's sieve in the form described, for example, in [1, Ch. 8], which is an implication of the Bonferroni inequalities.

Theorem 10: Let $B_{1}, \ldots, B_{n}$ be events and $Y$ be the number of $B_{i}$ that hold. Suppose that for any set $\left\{i_{1}, \ldots, i_{k}\right\} \subseteq\{1, \ldots, n\}$

$$
\operatorname{Pr}\left(B_{i_{1}} \wedge \cdots \wedge B_{i_{k}}\right)=\operatorname{Pr}\left(B_{1} \wedge \cdots \wedge B_{k}\right)
$$

and there is a constant $\mu$, so that for any fixed $k$

$$
n^{k} \operatorname{Pr}\left(B_{1} \wedge \cdots \wedge B_{k}\right) \sim \mu^{k} .
$$

Then $Y$ is also asymptotically Poisson with mean $\mu$.

For applying Theorem 10 , let $B_{i}$ be the event that $X_{i}$ is isolated for $1 \leq i \leq n$, and $Y$ be the number of $B_{i}$ 's that hold. Then $Y$ is exactly the number of isolated nodes. Similarly, let $B_{i}^{\prime}$ be the event that $X_{i}$ is isolated and active for $1 \leq i \leq n$, and $Y^{\prime}$ be the number of $B_{i}$ 's that hold. Then $Y^{\prime}$ is exactly the number of isolated active nodes. Obviously, for any set $\left\{i_{1}, \ldots, i_{k}\right\} \subseteq$ $\{1, \ldots, n\}$

$$
\begin{aligned}
& \operatorname{Pr}\left(B_{i_{1}} \wedge \cdots \wedge B_{i_{k}}\right)=\operatorname{Pr}\left(B_{1} \wedge \cdots \wedge B_{k}\right) \\
& \operatorname{Pr}\left(B_{i_{1}}^{\prime} \wedge \cdots \wedge B_{i_{k}}^{\prime}\right)=\operatorname{Pr}\left(B_{1}^{\prime} \wedge \cdots \wedge B_{k}^{\prime}\right) .
\end{aligned}
$$

In addition

$$
\operatorname{Pr}\left(B_{1}^{\prime} \wedge \cdots \wedge B_{k}^{\prime}\right)=p^{k} \operatorname{Pr}\left(B_{1} \wedge \cdots \wedge B_{k}\right) .
$$

Thus, in order to prove Theorem 9 , it suffices to show that if $r=\sqrt{(\ln n+\xi) / \pi p n}$ for some constant $\xi$, then for any fixed $k$

$$
n^{k} \operatorname{Pr}\left(B_{1} \wedge \cdots \wedge B_{k}\right) \sim e^{-k \xi} .
$$

The proof of this asymptotic equality will use the following two lemmas.

Lemma 11: For any $x \in \Omega$

$$
\operatorname{Pr}\left(B_{1} \mid X_{1}=x\right)=\left(1-p \nu_{r}(x)\right)^{n-1}
$$

Proof: For any $x \in \Omega$

$$
\begin{aligned}
\operatorname{Pr} & \left(B_{1} \mid X_{1}=x\right) \\
& =\operatorname{Pr}\left(\forall 2 \leq i \leq n, X_{i} \text { is either outside } \nu_{r}(x) \text { or inactive }\right) \\
& =\sum_{i=0}^{n-1} q^{i}\left(\begin{array}{c}
n-1 \\
i
\end{array}\right)\left(1-\nu_{r}(x)\right)^{n-1-i} \nu_{r}(x)^{i} \\
& =\left(1-\nu_{r}(x)+q \nu_{r}(x)\right)^{n-1}=\left(1-p \nu_{r}(x)\right)^{n-1} .
\end{aligned}
$$

Lemma 12: For any $k \geq 2$ and $\left(x_{1}, \ldots, x_{k}\right) \in \Omega^{k}$

$$
\begin{aligned}
\operatorname{Pr}\left(B_{1} \wedge \cdots \wedge B_{k} \mid X_{i}=x_{i}, \quad 1\right. & \leq i \leq k) \\
& \leq\left(1-p \nu_{r}\left(x_{1}, \ldots, x_{k}\right)\right)^{n-k}
\end{aligned}
$$

In addition, the equality is achieved for $\left(x_{1}, \ldots, x_{k}\right) \in C_{k k}$. 
Proof: For any $\left(x_{1}, \ldots, x_{k}\right) \in \Omega^{k}$

$$
\begin{aligned}
& \operatorname{Pr}\left(B_{1} \wedge \cdots \wedge B_{k} \mid X_{i}=x_{i}, \quad 1 \leq i \leq k\right) \\
& \leq \operatorname{Pr}\left(\nu_{r}\left(x_{1}, \ldots, x_{k}\right)\right. \text { contains no active node } \\
&\left.\quad \quad \text { in } X_{k+1}, \ldots, X_{n}\right) \\
&=\sum_{j=0}^{n-k} q^{j}\left(\begin{array}{c}
n-k \\
j
\end{array}\right)\left(1-\nu_{r}\left(x_{1}, \ldots, x_{k}\right)\right)^{n-k-j} \\
& \quad \cdot \nu_{r}\left(x_{1}, \ldots, x_{k}\right)^{j} \\
&=\left(1-p \nu_{r}\left(x_{1}, \ldots, x_{k}\right)\right)^{n-k} .
\end{aligned}
$$

For any $\left(x_{1}, \ldots, x_{k}\right) \in C_{k k}$

$$
\begin{aligned}
\operatorname{Pr} & \left(B_{1} \wedge \cdots \wedge B_{k} \mid X_{i}=x_{i}, \quad 1 \leq i \leq k\right) \\
= & \operatorname{Pr}\left(\forall 1 \leq i \leq k, \nu_{r}\left(x_{i}\right)\right. \text { contains no active node } \\
& \left.\quad \text { in } X_{k+1}, \ldots, X_{n}\right) \\
= & \sum_{m_{1}+\ldots+m_{k}=0}^{n-k} \operatorname{Pr}\left(\forall 1 \leq i \leq k, \nu_{r}\left(x_{i}\right) \text { contains } m_{i}\right. \\
& \quad \text { inactive nodes and no active nodes } \\
& \left.\quad \text { in } X_{k+1}, \ldots, X_{n}\right) \\
= & \sum_{m_{1}+\ldots+m_{k}=0}^{n-k}\left(\begin{array}{c}
n-k \\
m_{1}, \ldots, m_{k}
\end{array}\right)\left(\prod_{i=1}^{k}\left(q^{m_{i}} \nu_{r}\left(x_{i}\right)^{m_{i}}\right)\right) \\
& \cdot\left(1-\nu_{r}\left(x_{1}, \ldots, x_{k}\right)\right)^{n-k-\sum_{i=1}^{k} m_{i}} \\
= & \left(1-p \nu_{r}\left(x_{1}, \ldots, x_{k}\right)\right)^{n-k} .
\end{aligned}
$$

Now we are ready to prove the asymptotic equality (1). From Lemmas 11 and 5

$$
n \operatorname{Pr}\left(B_{1}\right)=n \int_{\Omega}\left(1-p \nu_{r}(x)\right)^{n-1} d x \sim e^{-\xi} .
$$

So the asymptotic equality (1) is true for $k=1$. Now we fix $k \geq 2$. From Lemmas 12, 6, and 7

$$
\begin{aligned}
& n^{k} \operatorname{Pr}\left(B_{1} \wedge \cdots \wedge B_{k} \text { and }\left(X_{1}, \ldots, X_{k}\right) \in \Omega^{k} \backslash C_{k k}\right) \\
& \leq n^{k} \int_{\Omega^{k} \backslash C_{k k}}\left(1-p \nu_{r}\left(x_{1}, \ldots, x_{k}\right)\right)^{n-k} \prod_{i=1}^{k} d x_{i}=o(1) .
\end{aligned}
$$

From Lemmas 12 and 8

$$
\begin{aligned}
n^{k} \operatorname{Pr}\left(B_{1}\right. & \left.\wedge \cdots \wedge B_{k} \text { and }\left(X_{1}, \ldots, X_{k}\right) \in C_{k k}\right) \\
& =n^{k} \int_{C_{k k}}\left(1-p \nu_{r}\left(x_{1}, \ldots, x_{k}\right)\right)^{n-k} \prod_{i=1}^{k} d x_{i} \sim e^{-k \xi} .
\end{aligned}
$$

Thus, the asymptotic equality (1) is also true for any fixed $k \geq 2$. This completes the proof of Theorem 9 .

\section{CONCLUSION}

Assume that a wireless ad hoc network consists of $n$ nodes which are independently and uniformly distributed in a unit-area disk, and become active independently with probability $p$ for some constant $0<p \leq 1$. Such a wireless ad hoc network can be modeled as a random geometric graph over Bernoulli nodes. We show that if all nodes have a maximum transmission radius $r_{n}=\sqrt{(\ln n+\xi) / \pi p n}$ for some constant $\xi$, then the total number of isolated nodes is asymptotically Poisson with mean $e^{-\xi}$, and the total number of isolated active nodes is also asymptotically Poisson with mean $p e^{-\xi}$. These asymptotic distributions will serve as the basis for our further probabilistic study on the connectivity of the active nodes.

A variant of the random geometric graphs studied in this paper is to replace the Bernoulli nodes model by the Bernoulli links model. In this variant, all nodes are assumed to be active, but all links may be active independently with probability $p$. It would be interesting to study the asymptotic distribution of the number of isolated nodes and asymptotic probability of the network being connected.

\section{REFERENCES}

[1] N. Alon and J. H. Spencer, The Probabilistic Method, 2nd ed. New York: Wiley, 2000.

[2] B. Bollobás, Random Graphs, 2nd ed. Cambridge, U.K.: Cambridge Univ. Press, Oct. 1, 2001

[3] H. Dette and N. Henze, "The limit distribution of the largest nearestneighbor link in the unit $d$-cube," J. Appl. Prob., vol. 26, pp. 67-80, 1989.

[4] P. Erdôs and A. Rényi, "On the evolution of random graphs," Pub. Math. Inst. Hungarian Acad. Sci., vol. 5, pp. 17-61, 1960.

[5] E. N. Gilbert, "Random plane networks," J. Soc. Ind. Appl. Math., vol. 9, no. 4, pp. 533-543, Dec. 1961.

[6] P. Gupta and P. R. Kumar, "Critical power for asymptotic connectivity in wireless networks," in Stochastic Analysis, Control, Optimization and Applications: A Volume in Honor of W. H. Fleming, W. M. McEneaney, G. Yin, and Q. Zhang, Eds. Boston, MA: Birkhauser, Mar. 1998, pp. 547-566.

[7] M. D. Penrose, "The longest edge of the random minimal spanning tree," Ann. Appl. Prob., vol. 7, no. 2, pp. 340-361, 1997.

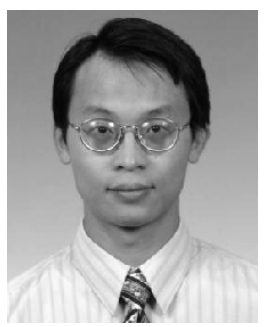

Chih-Wei Yi received the Ph.D. degree from the Illinois Institute of Technology, Chicago, and the M.S. and B.S. degrees from National Taiwan University, Taipei, Taiwan, R.O.C.

He is currently an Assistant Professor in Computer Science with the National Chiao Tung University, Hsinchu, Taiwan, R.O.C. His research focuses on wireless ad hoc and sensor networks.

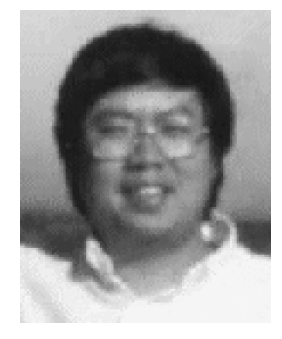

Peng-Jun Wan received the Ph.D. degree from the University of Minnesota, Minneapolis, the M.S. degree from The Chinese Academy of Science, Beijing, China, and the B.S. degree from Tsinghua University, Beijing, China.

$\mathrm{He}$ is currently an Associate Professor in Computer Science with the Illinois Institute of Technology, Chicago, and with City University of Hong Kong, Hong Kong. His research interests include wireless networks, optical networks, and algorithm design and analysis. 


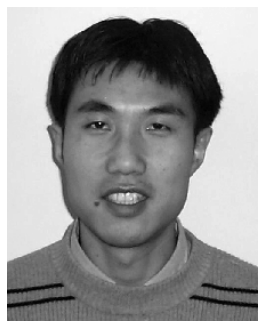

Xiang-Yang Li (M'00) received the M.S. and Ph.D. degrees in computer science from the University of Illinois at Urbana-Champaign in 2000 and 2001, respectively, and the B.S. degrees in computer science and business management from Tsinghua University, Beijing, China in 1995.

He has been an Assistant Professor of Computer Science with the Illinois Institute of Technology, Chicago, since 2000. His research interests span wireless ad hoc and sensor networks, noncooperative computing, computational geometry, optical networks, and cryptography. He has been a Guest Editor of special issues for ACM Mobile Networks and Applications and the IEEE JOURNAL ON SELECTED AREAS IN COMMUNICATIONS. He is a Member of the ACM.
Ophir Frieder (SM'93-F'02) is the IITRI Chair Professor of Computer Science and the Director of the Information Retrieval Laboratory at the Illinois Institute of Technology, Chicago. 